\title{
Mithridatism for dementia? Hypoxic - Hyperoxic training in dementia
}

\author{
Christos Tsagkaris', Rehab A. Rayan², Eleni Konstantara ${ }^{3}$, Lolita Matiashova ${ }^{4}$ Valeriia Danilchenko \\ 'University of Crete, Heraklion, Greece \\ ${ }^{2}$ Alexandria University, Alexandria, Egypt \\ ${ }^{3}$ Medical University of Sofia, Sofia, Bulgaria
}

${ }^{4}$ GI "L.T.Malaya Therapy National Institute of the NAMS of Ukraine, Kyiv, Ukraine

${ }^{5}$ Kharkiv Regional Perinatal Center, Department of Post-Intensive Care, Rehabilitation and Nursing of Premature Newborns, Kharkiv, Ukraine

\begin{abstract}
Introduction: Intense research on dementia has been conducted during the last years. As advances in the field have started changing the landscape of dementia treatment, it is necessary to assess the impact of novel therapeutic modalities.
\end{abstract}

Purpose: The current evidence about hypoxic - hyperoxic treatment for dementia is reviewed in this article.

Methods: We conducted a thorough PubMed/MEDLINE and Google Scholar search.

Results: Preclinical and clinical data are available. Hypoxic - hyperoxic treatment is encouraged in the context of the multimodal treatment of dementia. There are concerns about the recovery of memory with regard to specific modalities of this treatment. Future perspectives are highlighted in the light of potentially useful biomarkers and health policy.

Conclusion: While constant updates and further research is critical to understand the impact of hypoxic - hyperoxic treatment in dementia, the available studies are limited and, hence, research that is more extensive is necessary. Currently, it is important to assess the current state of knowledge highlighting the success but also the stalemates of this treatment.

\section{Keywords}

Intermittent hypoxia, Hypoxic - hyperoxic training, Cognitive performance, Dementia.

Address for correspondence:

Christos Tsagkaris, University of Crete, Faculty of Medicine, Heraklion, Greece, e-mail: chriss20x@gmail.com

This work is licensed under a Creative Commons Attribution-

Submitted for publication: 15 June 2020

NonCommercial 4.0 International License (CC BY-NC 4.0).

Received: 15 June 2020

(c) (7) (9)

(c) Copyright: Tsagkaris, Rayan, Konstantara, Matiashova, Danilchenko, 2020

Accepted for publication: 19

Licensee NDSAN (MFC- Coordinator of the NDSAN), Italy

DOI: http://doi.org/10.32437/mhgci.v3i1.82

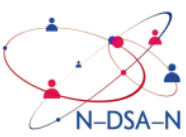




\section{Introduction}

The concept of Mithridatism dates back to Mithridates the 6th, king of Pontos during the 1st century BC. Mithridates would consume nonlethal amounts of poison to protect himself from poisoning in the future. By taking the poison in gradually increased doses, the king made himself "immune" enough to the poison to make it ineffective when he would attempt to take his life some years later after a defeat in battle. Mithridates would have to order one of his bodyguards to take his head eventually. In a vague sense, he managed to create tolerance against a toxic factor. (Valle et al., 2017)

The pattern of Mithridatism has been classic in toxicology and pharmacology throughout the years. Interestingly, hypoxic - hyperoxic treatment seems to abide by this concept. Essentially, hypoxic - hyperoxic treatment consists of the use of hypoxia, a genuinely toxic factor for neuronal integrity and memory, as a training stimulus to create tolerance against ischemia and safeguard human memory. Scientists have come to investigating such treatment modalities due to the prevalence and morbidity of neurocognitive disorders.

Neurocognitive disorders, especially major neurocognitive disorders (dementias), have serious effects on patients, families, the health system, and the economy (Hugo \& Ganguli, 2014). Alzheimer's disease (AD) is a major risk for mortality (Murphy et al., 2013), admission to hospitals and nursing facilities, and home healthcare in the United States (US). The expanses of health services and the informal expanses of non-paid caregiving of dementia patients' are high and escalating. Caregivers from the family suffer high affective pressure, depression among other health issues ("2020 Alzheimer's Disease Facts and Figures," 2020). Globally, In 2010, almost 35.6 million individuals were assumed to be surviving with dementia, a number anticipated to grow to about 115.4 million individuals by 2050 (Prince et al., 2013).

\section{Pathophysiology of dementia}

Amyloid plaques and neurofibrillary tangles are featured abnormalities, which determine AD. Amyloid plaques comprise mainly a 40-42 amino acid peptide called amyloid- $\beta$ (AB), which is accumulated in fibrils including a high $\beta$-sheet structure. Plaques turn insoluble and sediment in the brain's outside cell spaces. Amyloid plaques are usually linked to distended, dystrophic neurites, astrogliosis, and activated microglia that make a neuritic plaque. However, amyloid plaques and neurofibrillary tangles aggregate inside the cell in neurons. (Prince et al., 2013). A- $\beta$ is naturally created by neurons inside the brain and released in the brain's outside cell spaces where during the pathogenicity of $A D$ it shifts configuration, turns insoluble, and sediment as plaques. A- $\beta$ does not have an identified, physiologic role, yet an increasing evidence has shown that under specific testing circumstances, A- $\beta$ could regulate synaptic transmission. Yet, the function of $A-\beta$ in natural synaptic role or in the disease's context is unknown (Chavez et al., 2000).

The role of hypoxia in neurodegeneration and dementia

Hypoxia regulates metapolyzing amyloid plaque protein (APP), causing a growing production of $A \beta$ through the amyloidogenic mechanism. Time-reliant hypoxic upregulation of APP has been further proven at the mRNA and protein levels, following 10-180 mins of ischemia, which might function as a guarding pathway to raise the levels of neuroprotective soluble APP $\alpha$ (Serebrovska et al., 2019). Yet, most times the growing APP leads to more levels of $A \beta$, not soluble APP $\alpha$ since hypoxia prefers metapolizing APP through the amyloidogenic mechanism (Urike Bayer et al., 2017)

\section{Purpose}

To review the current evidence about Hypoxia - Hyperoxia treatment for dementia.

\section{Methodology}

This is a literature review study. We searched Pubmed and Google Scholar with the following strategy: ((prospective[Title/Abstract] OR cohort[Title/Abstract] OR follow-up[Title/Abstract] OR review[Title/Abstract] $\quad$ OR longitudinal[Title/Abstract] OR metaanalysis[Title/Abstract] OR systematic review[Title/Abstract]) AND (hypoxia - hyperoxia treatment[Title/Abstract] dementia[Title/Abstract])) AND (hypoxia OR hyperoxia OR dementia) AND \&quot; humans\&quot;[MeSH Terms], up until August 30 2020. This search strategy aims to identify: 1) Clinical trials involving hypoxia hyperoxia treatment; 2) Other original studies or metanalyses related to the use of this treatment. Original, peer-reviewed studies in English and Russian were included.

\section{Results}

There has been encouraging evidence from the field of basic research that supported the methods that led to their studying in clinical context. There are studies, both clinical and basic researches that show that intermittent hypoxic- 
hyperoxic treatment can reduce dementia and more specifically Alzheimer's disease (AD) or mild cognitive impairment which is a precursor of $A D$.

It is known that in $A D$ the pathological evidence is the presence of amyloid plagues. Clinical data has showed that is $A D$ patients the reduction of cerebral perfusion happens before memory and cognitive impairment. Hypoxia is the direct consequence of hypoperfusion. Improving oxygen supply in the brain might have a positive impact on AD pathology. Normobaric hyperoxia (NBO), not only provides more oxygen but was also found to be protective in recent experimental and clinical pilot studies. Morris water maze tests showed that NBO treatment improved the spatial learning and memory problems in A $A P P / P S 1$ transgenic mice. Immunohistochemical and thioflavin $S$ staining showed that NBO treatment significantly decreased $A \beta$ deposition and neuritic plaques formation in the cortex and hippocampus of AßPP/PS 1 transgenic mice. Immunoblotting and ELISA assay revealed that NBO treatment reduced $A \beta$ production by inhibiting $\gamma$-secretase cleavage of A PPP. From the above it is suggested that NBO may have a potential therapeutic effect at the early stages of AD. (Gao et al., 2011)

A study conducted by Malle et al. focused on the effects of normobaric hypoxia $(\mathrm{NH})$ exposure has on memory and physiology of the human body as well as the physiological and cognitive effects of oxygen breathing before and after the $\mathrm{NH}$ exposure.

For this study 86 healthy men divided randomly into 4 groups, were used. The groups were: the Normoxia-Air group ( $\mathrm{N}=23$ ), where subjects were breathing air, the Hypoxia-Air group ( $\mathrm{N}=$ 22), $\mathrm{NH}$ exposure was preceded and followed by air breathing, the Normoxia- $\mathrm{O}_{2}$ group $(\mathrm{N}=21$ ), similar to the Normoxia-Air group, except with the addition of $100 \% \mathrm{O}_{2}$ breathing periods and the Hypoxia- $\mathrm{O}_{2}$ group ( $\mathrm{N}=20$ ), whose participants were exposed to $100 \% \mathrm{O}_{2}$ before and after $\mathrm{NH}$ exposure. The Paced Auditory Serial Addition Test was performed to test their memory. Moreover, peripheral oxygen saturation $\left(\mathrm{Spo}_{2}\right)$, heart rate $(\mathrm{HR})$, and electroencephalogram (EEG) were recorded. (Malle et al., 2016)

The results from this study showed that acute $\mathrm{NH}$ exposure caused a typical physiological response like decreased $\mathrm{Spo}_{2}$ and increased HR, but not the same as the physiological response to acute hypobaric hypoxia. Impairment in working memory was also caused by the acute $\mathrm{NH}$. Oxygen breathing after $\mathrm{NH}$ exposure caused a slowing in the EEG which is associated with making working memory ability worse. For this reason, $\mathrm{NH}$ is suggested to be surrounded by air breathing. (Malle et al., 2016)

In another study, researchers used quantitative proton magnetic resonance spectroscopy to evaluate the regional metabolic alterations, after a 24-hour hypoxic or hyperoxic exposure on the background of ischemic brain insult, in a total of 60 female Wistar rats which were divided in two age-groups of rats of equal number: young - 3 months old and aged - 24 months old. Each age group was further subdivided into three subgroups of 10 rats each. Two of these subgroups were anesthetized with Nembutal (30 $\mathrm{mg} \cdot \mathrm{kg}-1$ ), after overnight fast, and by ligation of the right common carotid artery, cerebral ischemia was induced to them. After taking extracts from three different brain regions (fronto-parietal and occipital cortices and the hippocampus) from both hemispheres concentrations of eight metabolites (alanine, choline-containing compounds, total creatine, $y$-aminobutyric acid, glutamate, lactate, myo-inositol and $\mathrm{N}$ acetylaspartate) were measured. This showed that in the control normoxic condition, there were significant increases in lactate and myo-inositol concentrations in the hippocampus of the aged rats, in comparison with the young ones. In the ischemia-hypoxia condition, the most predominant changes in the brain metabolites were found in the hippocampal regions of both young and aged rats, but the effects were more evident in the aged animals. The ischemiahyperoxia procedure caused less changes in the brain metabolites, which may indicate more limited tissue damage. (Watanabe et al. 2019)

As it is already well-known, chronic hypoxia stimulates angiogenesis in brain and other tissues. Therapeutic IHT (intermittent hypoxic training) then, can improve the vascularity of the brain and prevent $A D$. When in hypoxia, cerebral angiogenesis starts by the transcription factor, hypoxia-inducible factor-1 (HIF-1) when genes with promoter regions containing hypoxic response elements, including the vascular endothelial growth factor (VEGF) gene, are activated. (Takashi et al. 2019)

There are also clinical studies that support these findings. More specifically, Urike Bayer et al. in a clinical study in 2017 studied thirty-four patients from the Geriatric Day Clinic aged between 64 and 92 years old who participated in a controlled trial. These patients received randomly MTP and IHHT (experimental group-EG) or MTP and placebo-breathing with machine face mask (experimental group-CG) in a doubleblind fashion. Before and after the 5- to 7-week intervention period (MTI + IHHT vs. MTI + ambient air), cognitive function was evaluated by the Dementia-Detection Test (DemTect), the 
Sunderland Clock-Drawing Test (CDT), and functional exercise capacity by the total distance of the 6-Minute Walk Test (6MWT). (Bayer et al., 2017)

Results from other studies showed that after MTI + IHHT was administered, DemTect showed important improvement (+16.7\% vs. $-0.39 \%, \mathrm{P}<$ 0,001 ) as well as the 6MWT with a larger increase in $E G$ than $C G(+24.1 \%$ vs. $+10.8 \%, P=.021)$. Furthermore, the CDT showed similar results with DemTect with an increase in EG but decrease in CG $(+10.7 \%$ vs. $-8 \%, P=0,031)$. Also, there was found to be a relation between the changes of the 6MWT, the DemTect and the CDT. The studies concluded that, IHHT is easy in application and well tolerated by geriatric patients up to 92 years and, helped in the improvement in cognitive function and exercise capacity in geriatric patients after MTI (CIRRITO \& HOLTZMAN, 2008) (Lall et al., 2019).

Bayer et al. in 2019 updated the previous study by performing some additional tests and including new results. Like before, she studied thirty-four patients (64-92 years old) who participated in the double-blind clinical trial. The patients took part in a 5-7 weeks lasting MTl (strength, endurance, balance, reaction, flexibility, coordination, and cognitive exercises) and performed IHHT (breathing 10-14\% oxygen for $4-7$ min followed by $2-4$ min $30-40 \%$ oxygen) in the Hypoxic Group (HG) or placebo treatment with ambient air in the Normoxic Group (NG). Before and after all treatments, mobility was assessed by the Tinetti Mobility Test (TMT), the Timed-Up-and-Go Test (TUG) and Barthel-Index, while perceived health was evaluated by one part of the EQ-5D Test, the EQ visual analogue scale (EQ VAS).

These tests showed that after the MTI plus IHHT or normoxia sessions, results of the TMT, TUG, Barthel Index and EQ-VAS revealed no significant difference between $H G$ and NG (Bayet et al., 2019)

Another study indicated that, IHHT added to MTI did not cause any additional improvements in patient's health and mobility compared to MTI alone (Pichiule \& Lamanna, 2002).

Serebrovska et al. also conducted a study in 2019 which examined the effects of intermittent hypoxic-hyperoxic training (IHHT) on elderly patients with mild cognitive impairment (MCl) which is a precursor of AD. The study used twentyone participants between 51 and 74 years of age which were divided into three groups: Healthy Control $(n=7), \mathrm{MCl}+$ Sham $(n=6)$, and $\mathrm{MCl}+\mathrm{HHT}(\mathrm{n}=8)$. IHHT was performed five times per week for three weeks which means a total of 15 sessions. Each IHHT session had four cycles of 5-min hypoxia (12\% $\mathrm{FIO} 2)$ and 3-min hyperoxia
(33\% FIO2). Cognitive parameters, $A \beta$ and amyloid precursor protein (APP) expression, microRNA 29, and long non-coding RNA in isolated platelets as well as NETs in peripheral blood were investigated. (Serebrovska et al., 2019)

The study found an initial decline in cognitive function indices in both $\mathrm{MCl}+$ Sham and $\mathrm{MCl}+\mathrm{HHT}$ groups and important connections between cognitive test scores and the levels of circulating biomarkers of $\mathrm{AD}$. $\mathrm{HHHT}$ resulted in the improvement in cognitive test scores, along with significant increase in APP ratio and decrease in $A \beta$ expression and NETs formation one day after the end of three-week IHHT. These effects on $A \beta$ expression and NETs formation remained more pronounced one month after IHHT. In conclusion, the results from this pilot study suggested a potential usage of $\mathrm{HH}$ T as a new therapy to improve cognitive function in pre-AD patients and slow down the development of AD (Serebrovska et al., 2019).

\section{Discussion}

In this review, we elaborated on hypoxic hyperoxic treatment in the context of dementia. We retrieved information from original studies spanning from preclinical to clinical research. The existing evidence seems to back the use of hypoxic - hyperoxic treatment, however there are few preclinical and clinical studies. With regard to mechanisms, the effects of hypoxia on the nervous system and the prevention or progression of dementia vary from study to study, indicating that the results also depend on the design of the studies.

A previous review study of Lall et al, concluded that hypoxia can prevent and treat $A D$ (Lall et al., 2019). Our review has reached the same conclusion with regard to improving the status of patients with AD but we cannot reach the conclusion that hypoxic - hyperoxic treatment can prevent $A D$ among healthy individuals. Another review has indicated that intermittent hypoxia has not always beneficial effects on patients with AD. Genetic traits might have contributed in the variability of the results (Manukhina et al., 2016). We have also reached the same conclusion.

The interplay between preclinical and clinical studies is also notable. Preclinical studies were conducted first encouraging the design of clinical interventions. With clinical studies initiated, basic research kept proceeding unraveling key information for the personalization of hypoxic hyperoxic treatment. Interestingly, in a study on 40 male mice where $40 \%$ oxygen with normal atmospheric pressure was utilized in the early 
stage of Alzheimer disease, there was a notable improvement in AßPP/PS1 transgenic mice after 1-2 months of treatment. The same treatment had no effect in wild-type mice. Such findings support the hypothesis of genetic interference that was suspected in clinical studies. Translational clinical studies may detect biomarkers determining the individuals who will benefit more from hypoxic - hyperoxic treatment.

In another study, normobaric hypoxia (NH) appeared to decrease $\gamma$-secretase cleavage of $A \beta P P$ and $A \beta$ in mice (Gao et al., 2011). Taking into account the involvement of these factors in the pathogenesis of dementia, and particularly $A D$, this finding indicates that $I H H T$ can hinder the further progression of the disease. Provided that large clinical studies verify these outcomes, hypoxic - hyperoxic treatment can be used as a dementia stabilizer, while cognitive training can improve the functionality and the quality of life of the patients.

The interplay of clinical and preclinical research is highlighted in the study of Marci et al. Their data indicated that hypoxia leads to cerebral ischemia resulting in the damage of the hippocampus-controlled functions (Macri et al., 2010) and then a study on healthy young men verified that $\mathrm{NH}$ and hypobaric hypoxia $(\mathrm{HH})$ can have adverse effects on memory. In both cases, the damage was attributed to physiological response to acute $\mathrm{NH}$ and $\mathrm{HH}$. These modalities are different and using oxygen after acute $\mathrm{NH}$ can slow cerebral activity down harming the recovery of memory (Malle et al., 2016). This finding, a synergy between preclinical and clinical research, suggests a serious adverse effect of this treatment. Participants of future studies should be properly warned and monitored.

Other studies have identified further biomarkers in patients with $A D$. Exercise capacity, cognitive performance and safety in geriatric patients have been correlated with an increase in APP130 and APP110 fractions in platelets, decrease in $A \beta$ expression and downregulation of InCRNA BACE-AS and NETs formation (Serebrovska et al., 2019) (U. Bayer et al., 2017).

Hypoxic - hyperoxic treatment and contemporary healthcare

The early diagnosis of dementia spectrum diseases is a challenge for modern biomedicine. When it comes to hypoxic - hyperoxic treatment, the necessary assessment and monitoring of patients or healthy individuals undergoing such interventions will require improved imaging techniques. Watanabe et al. have suggested the visualization of $A 2$ noradrenergic neurons with MRI based on the detection of noradrenaline groups of cells in the brain by $\mathrm{Tl}$-weighted MRI with magnetization transfer (Watanabe et al., 2019).

Hypoxic - hyperoxic treatment would face cost and implementation issues. With small studies, absence of long-term results and a need for expensive additional genetic testing, Hypoxic hyperoxic treatment can be expensive and inaccessible to patients in the future. Regulatory and legal parameters are also implicated. University hospitals and centers of excellence can offer this treatment to large number of patients to verify its efficacy and define the eligible population. If this intervention proves to be cost effective, the necessary regulatory steps can be taken. Licensing procedures ought to take into account the multimodal approach of providing this treatment.

\section{Limitations of the study}

Currently it seems that preclinical and clinical evidence regarding hypoxic - hyperoxic treatment is encouraging but limited. Systematic approaches on studies with small populations or short follow up time would not lead to credible conclusions. Future studies will need to study the effect of hypoxic - hyperoxic treatment in larger population sets. On the other hand, the indication that hereditary traits might affect the efficacy of hypoxic - hyperoxic treatment represents an opportunity of tracking genetic biomarkers. In this context hypoxic - hyperoxic treatment could be reserved as a precision medicine modality in the future.

\section{Conclusions}

In the existing studies, hypoxic - hyperoxic treatment appears as a beneficial additional treatment for dementia. It may contribute in preventing dementia in healthy individuals. It seems that genetic factors are involved in the efficacy of the treatment. Normobaric and hyperbaric hypoxia modalities can be used in patients with damage of the nervous system, which leads to benefits in cognitive function, however depending on the administration of hypoxic - hyperoxic treatment memory recovery may be impaired especially in healthy individuals (Serebrovska et al., 2019) (Malle et al., 2016). Mithridates has managed to determine the optimal way of self-poisoning to avoid adverse effects. Nowadays, the same challenge falls upon contemporary scientists with regard to the use of hypoxic - hyperoxic treatment in dementia.

Conflict of interest 
The authors declare no conflict of interest with regard to this study.

\section{References}

2020 Alzheimer's disease facts and figures. (2020). Alzheimer's and Dementia. https://doi.org/10.1002/alz. 12068

Bayer, U., Glazachev, O. S., Likar, R., Burtscher,

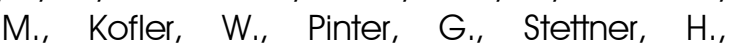
Demschar, S., Trummer, B., \& Neuwersch, S. (2017). Adaptation to intermittent hypoxiahyperoxia improves cognitive performance and exercise tolerance in elderly. Advances in Gerontology $=$ Uspekhi Gerontologii. https://doi.org/10.5281/zenodo. 1997725

Bayer, Urike, Likar, R., Pinter, G., Stettner, H., Demschar, S., Trummer, B., Neuwersch, S., Glazachev, O., \& Burtscher, M. (2017). Intermittent hypoxic-hyperoxic training on cognitive performance in geriatric patients. Alzheimer's and Dementia: Translational Research and Clinical Interventions. https://doi.org/10.1016/j.trci.201 7.01.002

Chavez, J. C., Agani, F., Pichiule, P., \& LaManna, J. C. (2000). Expression of hypoxia-inducible factor- $1 \alpha$ in the brain of rats during chronic hypoxia. Journal of Applied Physiology. https://doi.org/10.1 152/jappl.2000.89.5.1937

CIRRITO, J. R., \& HOLTZMAN, D. M. (2008). ANTIAMYLOID- $\beta$ IMMUNOTHERAPY AS A TREATMENT FOR ALZHEIMER'S DISEASE. In CNS Regeneration (pp. 295-318). Elsevier. https://doi.org/10.1016/B978-0123739940.50014-2

Gao, B., Long, Z., Zhao, L., \& He, G. (2011). Effect of normobaric hyperoxia on behavioral deficits and neuropathology in alzheimer's disease mouse model. Journal of Alzheimer's Disease. $\quad$ https://doi.org/10.3233/JAD-2011110308

Hugo, J., \& Ganguli, M. (2014). Dementia and Cognitive Impairment. Epidemiology, Diagnosis, and Treatment. In Clinics in Geriatric Medicine. https://doi.org/10.1016/..cger.2014.04.001

Lall, R., Mohammed, R., \& Ojha, U. (2019). What are the links between hypoxia and alzheimer's disease? In Neuropsychiatric Disease and Treatment. https://doi.org/10.2147/NDT.S203103

Macri, M. A., D'Alessandro, N., Di Giulio, C., Di Iorio, P., Di Luzio, S., Giuliani, P., Esposito, E., \& Pokorski, M. (2010). Region-specific effects on brain metabolites of hypoxia and hyperoxia overlaid on cerebral ischemia in young and old rats: a quantitative proton magnetic resonance spectroscopy study. Journal of
Biomedical

Science.

https://doi.org/10.1 186/1423-0127-17-14

Malle, C., Bourrilhon, C., Pierard, C., Quinette, P., Laisney, M., \& Eustache, F. (2016). Physiological and Cognitive Effects of Acute Normobaric Hypoxia and Modulations from Oxygen Breathing. Aerospace Medicine and Human Performance. https://doi.org/10.3357/AMHP.4335.2016

Manukhina, E. B., Downey, H. F., Shi, X., \& Mallet, R. T. (2016). Intermittent hypoxia training protects cerebrovascular function in Alzheimer's disease. Experimental Biology and Medicine. https://doi.org/10.1177/1535370216649060

Murphy, S. L., Xu, J., \& Kochanek, K. D. (2013). Deaths: final data for 2010. National Vital Statistics Reports : From the Centers for Disease Control and Prevention, National Center for Health Statistics, National Vital Statistics System, $61(4)$, $1-117$. https://pubmed.ncbi.nlm.nih.gov/24979972/

Pichiule, P., \& Lamanna, J. C. (2002). Angiopoietin-2 and rat brain capillary remodeling during adaptation and deadaptation to prolonged mild hypoxia. Journal of Applied Physiology. https://doi.org/10.1 152/japplphysiol.00318.200 2

Prince, M., Bryce, R., Albanese, E., Wimo, A., Ribeiro, W., \& Ferri, C. P. (2013). The global prevalence of dementia: A systematic review and metaanalysis. In Alzheimer's and Dementia

https://doi.org/10.1016/j.jalz.2012.11.007

Serebrovska, Z. O., Serebrovska, T. V., Kholin, V. A., Tumanovska, L. V., Shysh, A. M., Pashevin, D. A., Goncharov, S. V., Stroy, D., Grib, O. N., Shatylo, V. B., Bachinskaya, N. Y., Egorov, E., $X i$, L., \& Dosenko, V. E. (2019). Intermittent hypoxia-hyperoxia training improves cognitive function and decreases circulating biomarkers of Alzheimer's disease in patients with mild cognitive impairment: A pilot study. International Journal of Molecular Sciences. https://doi.org/10.3390/ijms20215405

Valle, G., Carmignani, M., Stanislao, M., Facciorusso, A., \& Volpe, A. R. (2017). Mithridates $\mathrm{VI}$ eupator king of pontus and the venomous snakes. In Central European Journal of Urology. https://doi.org/10.5173/ceju.2017.1479

Watanabe, T., Tan, Z., Wang, X., MartinezHernandez, A., \& Frahm, J. (2019). Magnetic resonance imaging of noradrenergic neurons. Brain Structure and Function. https://doi.org/10.1007/s00429-019-01858-0 